\title{
From Other Journals: A Review of Recent Articles by Our Editorial Team
}

\author{
Tarek Alsaied ${ }^{1,2}$. $\cdot$ Awais Ashfaq $^{3}$ \\ Accepted: 3 September 2021 / Published online: 13 September 2021 \\ (c) The Author(s), under exclusive licence to Springer Science+Business Media, LLC, part of Springer Nature 2021
}

\begin{abstract}
In this review we provide a brief description of recently published articles addressing topics relevant to pediatric cardiologists. Our hope is to provide a summary of the latest articles published recently in other journals in our field. The articles address (1) a summary of a scientific statement of the American Heart Association for diagnosis and treatment of myocarditis, (2) development of a perioperative risk score for in-hospital mortality after cardiac surgery in adults with congenital heart disease, (3) using a machine learning algorithm to predict cardiopulmonary deterioration in patients in the interstage period 1-2 $\mathrm{h}$ in advance using hospital monitor generated data, (4) risk factors for reoperation after the arterial switch operation, (5) the effect of mitochondrial transplantation for cardiogenic shock in pediatric patients, (6) comparing outcomes of primary or staged repair in tetralogy of Fallot with pulmonary atresia.
\end{abstract}

Keywords Heart transplantation · Fontan · COVID-19 · Collaterals · Tetralogy of Fallot · Norwood · Atrioventricular septal defect $\cdot$ Atrioventricular canal

In this review we provide a brief description of recently published articles addressing topics relevant to pediatric cardiologists. Our hope is to provide a summary of the latest articles published in other journals in our field [1].

Myocarditis remains challenging to diagnose, especially in children. Historically endomyocardial biopsy was the test of choice using histopathology-based diagnostic criteria such as the Dallas criteria. However, due to the invasive nature of endomyocardial biopsy and the patchy nature of myocarditis, clinical diagnosis was widely used until the last decade. The recent advances in tissue characterization by cardiac MRI, resulted in increased use of cardiac MRI to diagnose myocarditis in adults. The American Heart Association asked a group of experts to systematically review the available literature on diagnosing and managing myocarditis in children. For diagnosis, the statement suggested simplified framework (Fig. 3 in the manuscript). If the patient is having

Tarek Alsaied

tarek.alsaied84@gmail.com

1 Pittsburgh Children's Hospital Medical Center, Heart Institute, 3333 Burnet Avenue, Pittsburgh, PA, USA

2 Department of Pediatrics, University of Pittsburgh, Pittsburgh, PA, USA

3 Heart Institute, Johns Hopkins All Children's All Children's Hospital, St. Petersburg, FL, USA symptoms of heart failure or new onset systolic dysfunction with additional clinical features of myocarditis including LV dysfunction, elevated troponin, ventricular arrhythmia, chest pain, ST segment changes, and viral prodrome, then they recommended proceeding with confirmation testing. Confirmation testing depends on patient stability, ability to sedate, and institutional technical capabilities. CMR will be a next step to confirm the diagnosis and if the diagnosis requires a different modality, a biopsy maybe considered. It is important to combine the findings from the testing modalities and the clinical picture of the patients. Cardiac MRI will be able to detect edema with increased $\mathrm{T} 2$ time and fibrosis as seen with an increase in the $\mathrm{T} 1$ time and the extracellular volume. Additionally, late gadolinium enhancement is common in the subepicardial area. It is important to note that patients with myocarditis can deteriorate quickly. Thus, these patients should be admitted to a floor that can initiate therapies quickly and ideally in a center that can provide mechanical support if needed. Antiviral therapy should be administered if an active viral infection is found. Immunomodulatory and immunosuppressive therapy is center specific, and each center should have their own protocol for treatment given the lack of definite data. The authors hope that this statement is not just an educational opportunity but also a unifying call to organize our efforts to better understand and treat this important pediatric disease [2]. 
Although rare, the in-hospital mortality is a feared complication following cardiac surgery in adult with congenital heart disease (ACHD). This study developed and validated a risk score that can be helpful to optimize risk assessment in this population. The study assessed the performance of previously used risk scores including EuroSCORE II components and procedure-related Adult Congenital Heart Surgery (ACHS) score. The study evaluated perioperative survival in patients aged $>16$ years undergoing congenital heart surgery in a large tertiary center between 2003 and 2019 in the United Kingdom. A risk variable-derived PEACH (PErioperative ACHd) score was calculated for each patient. Internal and external validation of the model was undertaken, including testing in a validation cohort of patients operated in a second European ACHD center in Milan between 2003 and 2015. Each variable was tested by univariate analysis to check the association with mortality. The variable was excluded if it does not contribute to the area under the curve to predict the mortality. A stepwise multivariable analysis was used. The final PEACH score included one point for each of the following factors: New York Heart Association class III-IV, more than 2 previous sternotomies, glomerular filtration rate $<60$, history of Fontan or CABG surgery, urgent surgery, active endocarditis, and hemoglobin below 100 or above $200 \mathrm{~g} / \mathrm{l}$.

The development cohort included 1782 procedures. Resternotomy was performed in 897 (50.3\%). There were 31 $(1.7 \%)$ in-hospital deaths which is a relatively low number. The PEACH score showed excellent discrimination ability (area under the curve [AUC]: 0.88; 95\% CI: 0.83-0.94), and performed better than the ACHS score (ACHS AUC: 0.69; 95\% CI: $0.6-0.78 ; \mathrm{P}=0.0003)$. The authors then created a simple 3-tiered risk stratification was formed: PEACH score 0 (in-hospital mortality $0.2 \%$ ), 1-2 (in-hospital mortality $3.6 \%$ ), and $\geq 3$ (in-hospital mortality $17.2 \%$ ). In a validation cohort of 975 procedures, the PEACH score retained its discriminative ability (AUC: 0.75 ; 95\% CI: 0.72-0.77). There was agreement in expected and observed perioperative mortality between cohorts in the creation and validation score. The author concluded that the PEACH is a simple score to predict in-hospital mortality for the ACHD population undergoing congenital heart surgery.

This score can help counsel adults with congenital heart disease who are expected to have surgery about the feared complication of in-hospital mortality. The associated editorial wondered if earlier intervention may be indicated before the development of worsening functional status and end organ injury [3].

Despite the improvement in survival, there is high mortality in the interstage period between stage I and stage II in patients with single-ventricle palliative surgery. Many of these events happen during the hospitalization while the patient is on continuous cardiorespiratory monitoring. The objective of this study was to develop and validate a realtime computer algorithm that can automatically recognize physiological precursors of cardiorespiratory deterioration in children with single-ventricle physiology during their interstage hospitalization. This was a retrospective study that was conducted from prospectively collected physiological data of subjects with single-ventricle physiology. Deterioration events were defined as a cardiac arrest requiring cardiopulmonary resuscitation (CPR) or an unplanned intubation. Physiological metrics were derived from the electrocardiogram (heart rate, heart rate variability, ST-segment elevation, and ST-segment variability, premature ventricular contractions) and the pulse oximeter (peripheral oxygen saturation and pleth variability index) from the monitors. A logistic regression model was trained to separate the physiological dynamics of the pre-deterioration phase from all other data generated by the study subjects. Data were split $50 / 50$ into model training and validation sets to enable independent model validation for the statistical model. The cohort consisted of 238 subjects admitted to the cardiac intensive care unit and stepdown units of Texas Children's Hospital over a period of 6 years. Approximately 300,000 h of high-resolution physiological waveform and vital sign data were collected using the Sickbay software platform (Medical Informatics Corp., Houston, Texas) in both the intensive care unit and the step-down unit. The physiological data generated by subjects were separated into 2 classes: study data (pre-deterioration) and control data (non-deterioration). A total of 112 cardiorespiratory deterioration events were evaluated in 72 subjects ( 45 cardiac deterioration events and 67 respiratory deterioration events). Ninety percent of the event happened in the intensive care unit. The risk index metric generated by the optimized algorithm was found to be sensitive and specific for detecting impending events $1-2 \mathrm{~h}$ in advance of overt extremis (receiver-operating characteristic curve area: $0.958 ; 95 \%$ confidence interval: $0.950-0.965)$. The model performed equally well in the validation cohort. The algorithm can provide $1-2 \mathrm{~h}$ of advanced warning for $62 \%$ of all cardiorespiratory deterioration events in children with single-ventricle physiology during their interstage period, with only 1 alarm being generated at the bedside per patient per day. The physiological patterns observed in the $1-2 \mathrm{~h}$ pre-deterioration phase are different from those at any other time during the interstage hospitalization of these patients. These patterns can be detected without human intervention using a trained machine learning algorithm with low number of false alarms. A multicenter trial is in progress for further validation and outcome testing for this machine learning algorithm. This early identification may provide a window of opportunity to intervene and prevent cardiopulmonary deterioration in this high-risk patient population [4] (Table 1).

The arterial switch operation (ASO) has changed the way how we care for patients with D-transposition of the 
Table 1 Summary of the 6 studies included in this review

\begin{tabular}{ll}
\hline Author & Study Summary \\
\hline Law et al.[1] & Diagnosis and management of myocarditis in children a scientific statement from the American Heart Association \\
& Myocarditis remains challenging to diagnose, especially in children \\
& Historically endomyocardial biopsy was the test of choice \\
& If the patient is having symptoms of heart failure or new onset systolic dysfunction with additional clinical features of \\
& myocarditis including LV dysfunction, elevated troponin, ventricular arrhythmia, chest pain, ST segment changes, \\
& and viral prodrome, then the authors recommended proceeding with confirmation testing including CMR and rarely \\
& biopsy. Immunomodulatory and immunosuppressive therapy is center specific, and each center should have their own \\
protocol for treatment given the lack of definite data & \\
This statement is a unifying call to organize our efforts to better understand and treat this important pediatric disease
\end{tabular}

Constantin et al. [2] Enhanced Assessment of Perioperative Mortality Risk in Adults With Congenital Heart Disease (ACHD)

This study developed and validated a risk score PEACH (PErioperative ACHd) for in-hospital mortality following surgery in adults with congenital heart disease

1782 procedure for creation with $1.7 \%$ mortality and 975 for validation at two major European centers

The score included one point for each of the following factors: New York Heart Association class III-IV, more than 2 previous sternotomies, glomerular filtration rate $<60$, history of Fontan or CABG surgery, urgent surgery, active endocarditis, and hemoglobin below 100 or above $200 \mathrm{~g} / \mathrm{l}$

The score showed excellent discrimination ability (area under the curve [AUC]: 0.88) PEACH score 0 (in-hospital mortality $0.2 \%$ ), 1-2 (in-hospital mortality $3.6 \%$ ), and $\geq 3$ (in-hospital mortality $17.2 \%$ )

The PEACH is a simple score to predict in-hospital mortality for the ACHD population undergoing congenital heart surgery

Rusin et al. ([3] Automated Prediction of Cardiorespiratory Deterioration in Patients With Single Ventricle

This study developed and validated a real-time computer algorithm that can automatically recognize physiological precursors of cardiorespiratory deterioration in children with single-ventricle physiology during their interstage hospitalization Deterioration events were defined as a cardiac arrest requiring cardiopulmonary resuscitation (CPR) or an unplanned intubation

Physiological metrics were derived from the electrocardiogram (heart rate, heart rate variability, ST-segment elevation, and ST-segment variability, premature ventricular contractions) and the pulse oximeter (peripheral oxygen saturation and pleth variability index) from the monitors

238 subjects admitted to the cardiac intensive care unit and stepdown units

A total of 112 cardiorespiratory deterioration events were evaluated in 72 subjects ( 45 cardiac deterioration events and 67 respiratory deterioration events)

The risk index metric generated by the machine learning algorithm was found to be sensitive and specific for detecting impending events $1-2 \mathrm{~h}$ in advance of overt extremis. The algorithm can provide $1-2 \mathrm{~h}$ of advanced warning for $62 \%$ of all cardiorespiratory deterioration events in children with single-ventricle physiology during their interstage period, with only 1 alarm being generated at the bedside per patient per day

This early identification may provide a window of opportunity to intervene and prevent cardiopulmonary deterioration in this high-risk patient population

Patel et al. [4] Risk factors for reoperation after arterial switch operation

403 patients studied from 1986 to 2017

Median follow-up was 8.6 years

Most common right-sided reoperations

Pulmonary arterioplasty $(n=11,2.7 \%)$ at 3.3 years

Supravalvar right ventricular outflow tract reconstruction (RVOTR) $(\mathrm{n}=9,2.2 \%)$ at 2.5 years

Most common left-sided reoperations

Aortic valve repair or replacement $(\mathrm{AVR} / \mathrm{r})(n=7,1.7 \%)$ at 13.6 years

Aortic root replacement (ARR) $(n=5,1.2 \%)$ at 13.6 years

Coronary Artery Bypass Graft/coronary patch arterioplasty $(n=5,1.2 \%)$ at 11.3 years

Taussig-Bing anomaly was a risk factor for any reoperation $(P=0.034)$

isk factors for specific reoperations

Ventricular septal defect for AVR/r $(P=0.038)$

Taussig-Bing anomaly for RVOTR $(P=0.004)$

Pulmonary artery banding for ARR $(P=0.028)$

Certain anatomic subsets carry different risks for late reoperation, and pulmonary artery and/or RVOT reinterventions tend to occur sooner than aortic reinterventions 
Table 1 (continued)

\begin{tabular}{|c|c|}
\hline Author & Study Summary \\
\hline Guariento et al.[5] & $\begin{array}{l}\text { Autologous mitochondrial transplantation for cardiogenic shock in pediatric patients following ischemia-reperfusion injury } \\
24 \text { patients included (MT, } n=10 \text {; Control, } n=14) \text { over a span of } 16 \text { years } \\
\text { Markers of systemic inflammatory response and organ function measured } 1 \text { day before and } 7 \text { days following revasculariza- } \\
\text { tion did not differ between groups } \\
\text { Successful separation from ECMO } \\
\text { MT group }(n=8,80 \%) \\
\text { Control group }(n=4,29 \%)(=0.02) \\
\text { Median circumferential strain immediately following IRI but before therapy was not significantly different between groups } \\
\text { Immediately following separation from ECMO, ventricular strain was significantly better in the MT group ( }-23.0 \% \text {; range, } \\
-20.0 \% \text { to }-28.8 \%) \text { compared with the Control group }(-16.8 \% \text {; range, }-13.0 \% \text { to }-18.4 \%)(P=0.03) \\
\text { Median time to functional recovery after revascularization was significantly shorter in the MT group ( } 2 \text { days vs } 9 \text { days; } \\
P=.02) \\
\text { Cox regression analysis showed higher composite estimated risk of cardiovascular events in the Control group (hazard } \\
\text { ratio, } 4.6 ; 95 \% \text { confidence interval, } 1.0 \text { to } 20.9 ; P=0.04) \\
\text { MT was associated with successful separation from ECMO and enhanced ventricular strain in patients requiring postcardi- } \\
\text { otomy ECMO for severe refractory cardiogenic shock after IRI }\end{array}$ \\
\hline Kim et al. [6] & $\begin{array}{l}\text { Primary versus staged repair in neonates with pulmonary atresia and ventricular septal defect } \\
\text { Comparison of initial palliation with BTS followed by second stage repair versus primary biventricular repair } \\
66 \text { neonates (BTS group: } n[30,45.5 \%) \text { or primary biventricular repair (pBVR group: } \mathrm{n}[36,54.5 \%) \\
\text { Overall mean follow-up duration was } 7.51 \pm 4.35 \text { years } \\
\text { 10-year overall survival was } 84.8 \% \text { ( } 94.4 \% \text { for pBVR vs } 75.7 \% \text { for BTS, P }[0.032) \\
\text { BTS group, } 2 \text { early and } 6 \text { interstage mortalities, and the pBVR group had no early and } 2 \text { late mortalities } \\
\text { In univariable analysis, genetic or extracardiac anomalies were a risk factor for mortality (HR, 5.56; P [0.038) } \\
\text { After achieving BVR, the pBVR group underwent significantly more frequent right ventricle outflow tract reinterventions } \\
(P<.001) \text { at a much earlier period ( } P \text { [0.017) compared with the BTS group } \\
\text { In neonates with ductal-dependent pulmonary atresia and ventricular septal defect, the primary BVR approach provides an } \\
\text { excellent survival rate, but the burden of right ventricle outflow tract reintervention is heavy. The staged approach with } \\
\text { BTS promotes pulmonary artery growth, but hospital and interstage mortality are significant }\end{array}$ \\
\hline
\end{tabular}

great arteries and operative mortality has improved significantly. As patients grow older, some require reoperations for right- or left-sided lesion. In this study, the authors explore the timing and the nature of those reoperations after ASO. This was a single-center retrospective study looking at 403 patients who underwent ASO from 1986 to 2017, divided into four eras: 1986 to $1989(n=34), 1990-1999(n=163)$, 2000-2009 ( $n=115)$ and 2010-2017 (n-91). Patients with Taussig-Bing anomaly, VSD, and arch abnormality were similar across eras. The majority of patients $(n=271,67 \%)$ had normal coronary artery anatomy, and the most common coronary anomaly was a circumflex artery arising from the right coronary artery $(n=64,22 \%)$.

Early mortality was $8 \%(n=32)$ for the entire cohort. For eras 1, 2, 3, and 4, early mortality was $24 \%(n=8), 9 \%$ $(n=14), 6 \%(n=7)$, and $3 \%(n=3)$, respectively $(P<0.001)$. The most common cause of early mortality was unrecoverable ventricular dysfunction postoperatively $(n=7)$, postoperative sepsis $(n=5)$, sudden postoperative cardiac arrest $(n=5)$, and pulmonary hypertensive crisis $(n=4)$. The distribution of cause of early mortality differed between eras with unrecoverable ventricular dysfunction and pulmonary hypertension being the most common causes in era 1 and postoperative stroke was the most common cause of early death in era 4 . An additional nine (2.4\%) patients have died during late follow-up and all died within 12 months of discharge.

Of the total 371 late survivors, the overall freedom from reoperation was $92 \%$. Of the patients who required reoperation, $29(8 \%)$ needed at least one reoperation, $6(1.8 \%)$ patients needed at least two reoperations, and $1(0.3 \%)$ needed three reoperations. The most common reoperations were pulmonic or right-sided interventions $(n=17,4.6 \%)$; aortic or left-sided interventions $(n=8,2.2 \%)$; coronary interventions ( $n=5,1.3 \%)$; and other $(n=7,1.9 \%)$. Rightsided interventions occurred sooner at 2.5 years (IQR: 1.4-5.7) postoperatively compared to left-sided interventions at 13.1 years (IQR: 10.1-15.5) postoperatively, $p=0.029$. Most common indication for the first left-sided intervention was aortic insufficiency (AI) without root aneurysm $(n=6,75 \%)$ and the most common indication for the first right-sided intervention was supravalvar pulmonary artery stenosis (SVPS; $n=8,47 \%$ ). The cumulative incidence of reoperation for the entire cohort was $6.9 \%, 13.1 \%$, and $13.1 \%$ at 10,20 , and 30 years, respectively. Survival without reoperation was $84 \%, 79 \%$, and $79 \%$ at 10,20 , and 30 years, respectively. Thirty-year cumulative incidence of any reoperation was $29 \%$ for Taussig-Bing anomaly patients and $11 \%$ for non-Taussig-Bing anomaly patients, $P<0.0001$ (Fig. 3). Twenty five-year cumulative incidence of coronary 
artery reoperation was $8 \%$ for coronary anomaly patients and $0.9 \%$ for normal coronary anatomy patients, $P=0.0143$.

On multivariable analysis, postoperative ECMO (OR: 40, CI: 7-218, $P<0.001$ ) and Taussig-Bing anomaly (OR: 3, CI: $1-11, P=0.034$ ) were found to be risk factors for any reoperation. For right-sided interventions, Taussig-Bing anomaly (OR: 7, CI: $2-36, P=0.011$ ) was found to be a risk factor for pulmonary valve intervention and for left-sided interventions, preoperative diagnosis of VSD (OR: 7, CI: 1-44, $P=0.038$ ) was a risk factor for aortic valve intervention, and preoperative pulmonary artery band (OR: 14, CI: $1-143$, $P=0.028)$ was a risk factor for aortic root intervention. The authors concluded that certain anatomic subsets carry different risks for late reoperation, and right-sided reinterventions occur sooner than left-sided interventions [5].

Myocardial ischemia-reperfusion injury (IRI) can result in cardiogenic shock despite revascularization. Clinical outcomes in pediatric patients remain poor, with the highest mortality rates or need for orthotopic heart transplantation (OHT) reported among patients requiring ECMO support. The authors have previously demonstrated that intramyocardial transplantation (MT) of autologous mitochondria is safe and associated with recovery of myocardial function [6].

This was a single-center retrospective study of patients requiring ECMO for postcardiotomy cardiogenic shock following IRI between May 2002 and December 2018. Postcardiotomy IRI was defined as coronary artery compromise detected by coronary angiography followed by successful revascularization. Patients undergoing revascularization and subsequent MT were compared with those undergoing revascularization alone. A sample of rectus abdominis muscle (6 $\mathrm{mm}$ in diameter) was used to harvest the mitochondria. Epicardial echocardiography was performed to determine the regions of left ventricular hypokinesis or akinesis and guide MT.

Twenty-four patients were included (MT, $\mathrm{n}=10$; Control, $n=14)$. Single-ventricle physiology was not different between groups. All patients experienced a myocardial ischemic event after cardiac surgery. Reperfusion was achieved by surgical revascularization $(n=15)$, percutaneous revascularization $(n=4)$, pharmacological $(n=2)$ patients. Markers of systemic inflammatory response such as white blood cell count, lactate and ionized calcium, and organ function measured 1 day before and 7 days following revascularization did not differ between groups. Successful separation from ECMO (defined as freedom from ECMO reinstitution within 1 week after initial separation) was possible for 8 patients in the MT group (80\%) and 4 in the Control group (29\%) $(P=0.02)$. Median circumferential strain immediately following IRI but before therapy was not significantly different between groups. Immediately following separation from ECMO, ventricular strain was significantly better in the MT group (23.0\%; range,
$20.0 \%-28.8 \%$ ) compared with the Control group (16.8\%; range, $13.0 \%-18.4 \%)(\mathrm{P}=0.03)$. Median time to functional recovery after revascularization was significantly shorter in the MT group ( 2 days vs 9 days; $\mathrm{P}=0.02$ ). There was no difference in median length of hospital stay, in-hospital mortality, or overall mortality between the groups. Cardiovascular events were lower in the MT group (20\% vs $79 \% ; P<0.01)$. Cox regression analysis showed higher composite estimated risk of cardiovascular events in the Control group (hazard ratio, 4.6; 95\% confidence interval, $1.0-20.9 ; P=0.04)$.

With these findings, the authors concluded that MT was associated with successful separation from ECMO and enhanced ventricular strain in patients with postcardiotomy cardiogenic shock needing ECMO [6].

Pulmonary atresia with ventricular septal defect (PA VSD) is a rare congenital cardiac anomaly that affects between 4.2 and 10 per 100,000 live births ([7]). The traditional surgical option for neonates with ductal-dependent PA VSD has been to perform initial palliation with a BlalockTaussig shunt (BTS) followed by a second stage biventricular repair (BVR) after the patient had grown. As operative techniques and perioperative management have advanced, however, early primary BVR has been adopted. In this study, the authors compare the practices at two major hospitals in South Korea involving staged versus primary repair.

Between 2004 and 2017, 66 neonates underwent surgery with palliative shunts (BTS group: $n=30,45.5 \%$ ) or primary biventricular repair (pBVR group: $n=36,54.5 \%$ ). Patients with significant MAPCAs were excluded. The 2 groups were similar in age, body weight, and Nakata index scores. The overall mean follow-up duration was $7.51 \pm 4.35$ years, and early and late results were compared between the groups.

The mean age at the time of the first operation was $18.05 \pm 7.71$ days, and the mean body weight was $3.23 \pm 0.46 \mathrm{~kg}$. The 10 -year overall survival was $84.8 \%$ (94.4\% for pBVR vs $75.7 \%$ for BTS, $P=0.032$ ). The BTS group had 2 early and 6 interstage mortalities, and the pBVR group had no early and 2 late mortalities. In the BTS group, concomitant angioplasty was performed in 17 patients $(56.7 \%)$. In the interstage period (mean duration $0.8 \pm 0.46$ years), 6 patients died due to cardiac arrest. The Nakata index score significantly increased during the interstage period $(P<0.001)$. Valved conduits were used in all but 4 patients. However, the pBVR group underwent total correction using RVOT patch and pericardial roll in 23 and 13 patients, respectively. Thirteen patients (36.1\%) underwent concomitant angioplasty. Overall survival at 1 and 10 years after the first operation was $87.9 \%$ and $84.8 \%$, respectively. The survival rates at 1 month, 1 year, and 10 years for each treatment group were $93.3 \%, 80 \%$, and $75.7 \%$ for the BTS vs. $100 \%, 94.4 \%$, and $94.4 \%$ for the pBVR group, respectively $(P=0.082)$. 
In the BTS group, the percentages of patients free from RVOT reintervention at 1 year, 2 years, and 5 years after BVR were $90.5 \%, 84.8 \%$, and $40.0 \%$, respectively. During a mean follow-up period of $5.07 \pm 3.75$ years, 6 percutaneous interventions and 8 reoperations were performed in 10 patients. On the other hand, the BVR group showed significantly less freedom from RVOT reintervention at 1 year, 2 years, and 5 years with values of $42.9 \%, 28.6 \%$, and $25.0 \%$, respectively. Thirty patients underwent 42 balloon angioplasties, 14 stent insertions, and 32 reoperations, and 53.3\% of the patients had more than 3 RVOT reinterventions. After achieving BVR, the pBVR group underwent significantly more frequent right ventricle outflow tract reinterventions $(P<0.001)$ at a much earlier period $(P=0.017)$ compared with the BTS group.

The authors concluded that in neonates with ductaldependent pulmonary atresia and ventricular septal defect, the primary BVR approach provides an excellent survival rate albeit with significant burden of right ventricle outflow tract reintervention. The staged approach with BTS promotes pulmonary artery growth, but hospital and interstage mortality are significant.

Authors' Contribution The author summarized the recent published literature in this review.

Funding Not applicable.

Data Availability Not applicable.

Code Availability Not applicable.

\section{Declarations}

Conflict of interest Not applicable.
Ethical Approval Not applicable.

Consent to Participate Not applicable.

Consent for Publication Not applicable.

\section{References}

1. Law YM, Lal AK, Chen S, Čiháková D, Cooper LT, Deshpande $S$ et al (2021) Diagnosis and management of myocarditis in children: a scientific statement from the American heart association. Circulation. https://doi.org/10.1161/CIR.0000000000001001

2. Constantine A, Costola G, Bianchi P, Chessa M, Giamberti A, Kempny A et al (2021) Enhanced assessment of perioperative mortality risk in adults with congenital heart disease. J Am Coll Cardiol 78(3):234-242

3. Rusin CG, Acosta SI, Vu EL, Ahmed M, Brady KM, Penny DJ (2021) Automated prediction of cardiorespiratory deterioration in patients with single ventricle. J Am Coll Cardiol 77(25):3184-3192

4. Patel PM, Herrmann JL, Bain E, Ladowski JM, Colgate C, Rodefeld MD et al (2021) Risk factors for reoperation after arterial switch operation. World J Pediatr Congenit Heart Surg 12(4):463-470

5. Guariento A, Piekarski BL, Doulamis IP, Blitzer D, Ferraro AM, Harrild DM et al (2021) Autologous mitochondrial transplantation for cardiogenic shock in pediatric patients following ischemiareperfusion injury. J Thorac Cardiovasc Surg 162(3):992-1001

6. Kim ER, Lee CH, Kim WH, Lim JH, Kim YJ, Min J et al (2021) Primary versus staged repair in neonates with pulmonary atresia and ventricular septal defect. Ann Thorac Surg 112(3):825-830

7. Egbe A, Uppu S, Lee S, Ho D, Srivastava S (2014) Changing prevalence of severe congenital heart disease: a population-based study. Pediatr Cardiol 35(7):1232-1238

Publisher's Note Springer Nature remains neutral with regard to jurisdictional claims in published maps and institutional affiliations. 